\title{
VORWORT DES AUTORS
}

Unser Land hat sich in einen mächtigen Industriestaat verwandelt. Die Zahl seiner Werke und Fabriken, seiner Eisenbahnen und Schächte vermehrt sich, seine Landwirtschaft wurde mechanisiert. Die schöpferische Arbeit von Millionen Sowjetmenschen, ausgerüstet mit der neuzeitlichen Technik, ist auf den Aufbau des Kommunismus gerichtet.

Die neuzeitliche Technik, ihr Aufbau und ihre Anwendung wird von einer großen Menge Berechnungen begleitet, welche in wissenschaftlichen Forschungsinstituten und Laboratorien, in Konstruktions- und Projektionsbüros, in den Werken, Schächten und auf dem Lande durchgeführt werden. Viele Leute sind mit Berechnungen beschäftigt, die enorm viel Zeit verschlingen. Manchmal erschweren komplizierte Berechnungen die Anwendung einer vollendeteren Technik, eine in die Breite gehende Einführung besserer Arbeitsmethoden.

Die wichtigste Aufgabe besteht darin, Methoden zu entwickeln, die, verbunden mit der Anwendung von Berechnungen, zum Gemeinbesitz der breitesten Massen werden, um Zehntausende qualifizierter Arbeiter von ermüdender und einförmiger Rechenarbeit zu befreien. Das bedeutet, neue Menschenreserven für unsere Volkswirtschaft freizumachen, Millionen Rubel zu sparen, Planung und Bau zu beschleunigen.

Eine große Rolle muß dabei die Nomographie spielen. Nomogramme sind die einfachsten Rechenhilfsmittel. Ihr Gebrauch ist bequem. Nach einem Nomogramm kann man die Lösung schnell ermitteln, die Zeitersparnis ist hierbei groß. Nomogramme erfordern auch keine besondere Qualifikation, um mit ihnen Berechnungen durchzuführen. In den Händen der Konstrukteure und Forscher finden die Nomogramme noch eine andere ganz wichtige Anwendung. Da die Nomogramme die Abhängigkeiten zwischen den gegebenen, durch Rechenformeln ausgedrückten Größen darstellen, gestatten sie, diese Abhängigkeiten anschaulich zu machen und neue physikalische Eigenschaften der Erscheinungen oder Eigenschaften von Konstruktionen $z u$ entdecken, die infolge der Kompliziertheit ihrer analytischen Form unentdeckt geblieben und nicht enträtselt worden wären. Ungeachtet des offensichtlichen Vorteils der nomographischen Methoden, werden diese verhältnismäßig selten und nicht wirksam genug angewandt. Dies erklärt sich einerseits durch die geringe Kenntnis, die breite Kreise von den Möglichkeiten der Nomographie haben; andererseits gibt die Mehrzahl der über Nomographie existierenden Leitfäden keine Antwort auf die grundlegenden Fragen der Praxis: wie gro $\beta$ ist die Genauigkeit bei Berechnungen an Nomogrammen, wie muß ein Nomogramm aufgebaut sein, um Berechnungen mit 
gegebener Genauigkeit durchzuführen, und wie kann man ein solches Nomogramm möglichst einfach konstruieren? Die Unfähigkeit, den Anwendungsbereich eines gegebenen Nomogramms zu bestimmen, führt oft zur Anwendung des Nomogramms auf Fälle, für die es nicht bestimmt ist; dies wiederum führt zu Mißverständnissen und zu Enttäuschungen bei der Verwendung nomographischer Methoden. Hier muß man auch die weitverbreitete irrige Meinung erwähnen, daß es unmöglich sei, Nomogramme für Berechnungen mit großer Genauigkeit anzuwenden.

Im vorliegenden Buch werden die Darstellungsmöglichkeiten mathematischer Formeln (analytischer Abhängigkeiten) durch Nomogramme und die Gebrauchsregeln verschiedener Nomogramme dargelegt.

Neben der Darlegung der allgemeinen Theorie der Nomogrammkonstruktionen wird in ihm eine systematische Analyse der Abhängigkeit zwischen der äußeren Form eines Nomogramms und der Ungenauigkeit der mit ihm erzielten Resultate durchgeführt; es werden auch die Methoden aufgezeigt, die eine Lösung mit gegebener Genauigkeit liefern. Dadurch unterscheidet sich dieses Buch bedeutend von anderen Büchern, die der Nomographie gewidmet sind. Das konkrete Herangehen an das Nomogramm, als eines Rechenhilfsmittels, das die Bedingungen seiner praktischen Bestimmung erfüllen muß, ist nur der sowjetischen Nomographie eigen. Diese Stellung der Nomographie wurde zum erstenmal von N.M. GERSEwanow in seinen "Grundlagen der Nomographie" klar umrissen und in dem Buch "Methodik der Konstruktion von Nomogrammen" von B. A. NEwsKI weiterentwickelt. Aber es war unmöglich, die dort niedergelegten Gedanken restlos auszunutzen, weil hinreichend wirksame Methoden der Konstruktion von Nomogrammen fehlten. Derartige Methoden sind in meinem Buch „Projektive Umwandlung von Nomogrammen" dargelegt, wo ein anschauliches Verfahren der Umwandlung von Nomogrammen angegeben wurde, das auf der Anwendung des ganz abstrakten Apparates der nichteuklidischen Geometrie beruht. Das vorliegende Buch ist die nächste Etappe in der aufgezeigten Richtung. In ihm sind sehr streng die Bedingungen formuliert, die Nomogramme erfüllen müssen; es werden die Methoden angegeben, nach denen auf der Grundlage der neu eingeführten Begriffe der "Charakteristik" einer Skala und ihrer besten Umformung ein Nomogramm obige Bedingungen erfüllen kann. Neben den Netzen für die projektive Umformung werden für die Nomogramme der Gleichungen dritter nomographischer Ordnung "Skelette" eingeführt, die die Durchführung sowohl projektiver als auch nichtprojektiver Umformungen dieser Nomogramme gestatten. Netze, Skelette, der Begriff von der Charakteristik und der besten Umformung bilden den Apparat, mit dessen Hilfe es möglich ist, vollendete Nomogramme aufzubauen, die unter den jetzigen Bedingungen so sehr gebraucht werden. Erhielt man mit den früher existierenden Methoden solche Nomogramme, so war dies eine Sache des Zufalls.

Das Buch besteht aus der Einführung, dem ersten Teil, der der Beschreibung von Fluchtlinientafeln und Netzen, ihrer Benutzungsanweisungen und der Theorie ihres Aufbaus gewidmet ist; dem zweiten Teil, der die Methoden der Konstruktion von Nomogrammen enthält, und $\operatorname{dem} \mathrm{Schlu} \beta$, wo die allgemeine Theorie der 
Darstellung funktionaler. Abhängigkeiten dargelegt ist, die die Nomogramme aller einzelnen Typen erfaßt. In den Text ist eine große Zahl (39) von Nomogrammen eingefügt, die so angelegt sind, wie es in der Ingenieurpraxis üblich ist. Sie können dem Unterricht als Hinweis über den Gebrauch von Nomogrammen dienen.

Man kann dieses Buch verschieden benutzen. Stellt man sich die Aufgabe, sich damit bekanntzumachen, was sind Nomogramme und wie gebraucht man sie, so genügt es, die Einführung, das erste Kapitel und die Paragraphen 19, 21, 22, 24, 25, 27, 28, 29 der folgenden Kapitel durchzulesen. Will sich der Leser eine Vorstellung über die Möglichkeiten der Nomographierung machen, dann muß er die Einführung, den ersten Teil des Buches und den Schluß durchlesen. Das Buch ist ganz darauf abgestellt, zu lehren, wie man für die einfachsten Fälle Nomogramme guter Qualität konstruiert, nachdem man sich mit Nomogrammen und ihrer Anwendung vertraut gemacht hat.

Das Gesagte bestimmt den Zweck des Buches. Man kann es als kurzes Lehrmittel der neuzeitlichen Nomographie betrachten. Das Buch ist bestimmt für Ingenieure, wissenschaftliche Arbeiter und Studenten der technischen Hochschulen. Die vorausgesetzten Kenntnisse, die zum Lesen des Buches notwendig sind, beschränken sich auf den Inhalt des Programms des ersten Semesters im Kurs der höheren Mathematik der technischen Hochschule. Die Darlegung der Theorie ist beschreibend. Dort, wo Beweise nötig wären, wurden Hinweise auf die entsprechende Literatur gemacht. Die Mehrzahl der Hinweise bezieht sich auf das Buch von N. A. GLAGolew: ,Die theoretischen Grundlagen der Nomographie", mit dem man sich bei einem weiteren Studium des Gegenstandes vertraut machen muß. 
